\title{
Acculturation, Depression, and Smoking Cessation: a trajectory pattern recognition approach
}

Sun S Kim ${ }^{1}$, Hua Fang ${ }^{2,4,5^{*}}$, Kunsook Bernstein ${ }^{3}$, Zhaoyang Zhang ${ }^{2}$, Joseph DiFranza ${ }^{2}$, Douglas Ziedonis ${ }^{6}$ and Jeroan Allison ${ }^{2}$

\begin{abstract}
Background: Korean Americans are known for a high smoking prevalence within the Asian American population. This study examined the effects of acculturation and depression on Korean Americans' smoking cessation and abstinence.

Methods: This is a secondary data analysis of a smoking cessation study that implemented eight weekly individualized counseling sessions of a culturally adapted cessation intervention for the treatment arm and a standard cognitive behavioral therapy for the comparison arm. Both arms also received nicotine patches for 8 weeks. A newly developed non-parametric trajectory pattern recognition model (MI-Fuzzy) was used to identify cognitive and behavioral response patterns to a smoking cessation intervention among 97 Korean American smokers (81 men and 16 women).

Results: Three distinctive response patterns were revealed: (a) Culturally Adapted (CA), since all identified members received the culturally adapted intervention; (b) More Bicultural (MB), for having higher scores of bicultural acculturation; and (c) Less Bicultural (LB), for having lower scores of bicultural acculturation. The CA smokers were those from the treatment arm, while MB and LB groups were from the comparison arm. The LB group differed in depression from the CA and MB groups and no difference was found between the CA and MB groups. Although depression did not directly affect 12-month prolonged abstinence, the LB group was most depressed and achieved the lowest rate of abstinence (LB: 1.03\%; MB: 5.15\%; CA: 21.65\%).
\end{abstract}

Conclusion: A culturally adaptive intervention should target Korean American smokers with a high level of depression and a low level of biculturalism to assist in their smoking cessation.

Trial registration: NCT01091363. Registered 21 March 2010.

Keywords: Culturally adaptive intervention, Trajectory pattern recognition, Multiple imputation, Fuzzy clustering, MIFuzzy, Longitudinal, Smoking cessation, Acculturation, Depression

\section{Background}

As per the 2014 Surgeon General Report, cigarette smoking caused more than 480,000 deaths in the United States each year between 2005 and 2009 [1]. The 2015 California Health Interview survey revealed a striking

\footnotetext{
*Correspondence: HFang2@umassd.edu; Hua.Fang@umassmed.edu; http://www.cis.umassd.edu/ fanglab/; http://www.umassmed.edu/fanglab/ 2University of Massachusetts Dartmouth and Medical School Dartmouth, Dartmouth, MA 02747, USA

${ }^{4}$ Department of Computer and Information Science, College of Engineering, University of Massachusetts Dartmouth, Dion Building, Room 317285 Old Westport Road Dartmouth, Dartmouth, MA 02747-2300, USA

Full list of author information is available at the end of the article
}

difference between non-Korean men and Korean men: $13 \%$ versus $34 \%$ respectively [2]. Reflecting the high prevalence rate, $71 \%$ of Korean men's cancer deaths in California were linked to smoking versus $30 \%$ for the general U.S. population [3, 4]. Furthermore, contrary to the decline observed among most gender and ethnic subgroups of Asian Americans, the prevalence of smoking among Korean American women has been on the rise (13-16\%) and now approaches that (14\%) of the general U.S. female population $[2,5]$.

There is an urgent call for smoking cessation interventions for Korean Americans. It has long been suggested 
that to be effective cessation programs need to be anchored in the norms, values, and experiences of cultural groups being studied [6-8]. Nevertheless, smoking cessation interventions developed for this ethnic group have been adapted at a surface level of the culture such as the adoption of common Asian cultural values (e.g., collectivism) and language concordance with Korean-speaking therapists [9-11]. These studies found a null or minimal treatment effect of an intervention compared to general health education or self-help materials. In contrast, a smoking cessation intervention developed at a deep level of Korean culture yielded an abstinence rate that was significantly higher than the rate from a standard cessation intervention [12].

Acculturation and depression have been frequently studied for their relationships with smoking and smoking cessation among Korean Americans. Especially, acculturation has been found to have a significant relationship with smoking, and gender moderates the relationship. Korean men with a low level of acculturation (e.g., newly arrived immigrants and limited English proficiency) are more likely to smoke than for those with a high level of acculturation (e.g., U.S.-born and proficient in English), whereas Korean women with high levels of acculturation are more likely to smoke than their counterparts [13, 14]. It was also reported that bicultural Korean men were least likely to be current smokers compared to traditional and acculturated Korean men while bicultural and acculturated Korean women were more likely to be current smokers than traditional Korean women [14]. The relationship between acculturation and smoking cessation was not much studied with Korean Americans. One study found an inverse relationship between acculturation and smoking cessation [15].

Depressive symptoms were found to have strong associations with smoking status. Current smokers consistently report higher depressive symptoms than former or never smokers [16, 17]. Similarly, among Asian Americans, current smokers reported higher depressive symptoms than non-smokers $[18,19]$. Depression is also a well-recognized barrier to immediate and longer-term smoking cessation [20]. Smokers with a history of depression (both major depression and dysthymia disorders) have a greater risk of relapse to smoking after a cessation attempt than smokers with no history of depression [20-22]. Nicotine withdrawal symptoms have been explained as a factor mediating the relationship between depression and smoking cessation. Smokers with a history of depression are likely to experience more withdrawal symptoms after a quit attempt and have a greater risk of relapse than smokers with no history [23].

The moderating effect of gender on the relationship between depression and smoking and between depression and smoking cessation has been reported although findings are inconsistent. It was found that female smokers were far more likely to be depressed than male smokers compared with the gender difference found among non-smokers [24, 25]. Similarly, a much stronger association between depression and smoking was found among Asian women than Asian men [26, 27]. For example, adjusted odds ratios of depression among Korean current smokers compared to Korean never smokers were 3.7 for women and 1.8 for men [27]. Strong gender-based smoking norm in Korea could be one of the contributing factors to the difference $[28,29]$. Korean women may internalize the negative portrait of a female smoker prevailing in Korean culture and become depressed as they continue to smoke. It was also reported that women had more depressive symptoms than men following quitting [30-32]. However, no study exists that examined any gender differences in the relationship between depression and smoking cessation among Asians or Asian Americans.

To the best of our knowledge, no study had examined the relationships between acculturation, depressive symptoms, and the latent patterns of cognitive responses to a multi-component culturally-adaptive cessation intervention, and whether the first two would predict treatment outcomes (abstinence vs. smoking) in Korean American smokers. Conventional approaches such as generalized linear mixed models and Cox regression models failed to identify any single baseline characteristic predictive of treatment outcomes in most cessation studies, due to substantial unexplained heterogeneity in smokers' responses to interventions. Thus, a newly developed trajectory pattern recognition model (MI-Fuzzy) was used to identify smokers' cognitive and behavioral response patterns over the period of interventions based on their psychological reactions to a culturally adapted cessation intervention and engagement with the intervention [33-36]. Conventionally, dichotomously assigned groups are used to represent a treatment arm that received a culturally adapted intervention (with a value of 1 ) and a comparison arm without cultural adaptation (with a value of 0 ) even though the intervention has multiple treatment components. This simple method cannot fully describe and capture cognitive and behavioral response variations in the culturally adapted and non-adapted interventions.

In addition, subgrouping (e.g., categorizing smokers based on demographical or baseline data) can generate spurious false-positive findings [37, 38]. During a longitudinal intervention, smokers usually display complex and varying behaviors such as relapsing or dropping out for various psychological, social and environmental reasons $[16,39-41]$. Their cognitive and behavioral variations during the intervention may contribute to different outcomes, herein, the rate of 12-month prolonged 
abstinence. Failure to appreciate these variations within, not only those between treatment and comparison arms, could ultimately lead to inappropriate assessment of the intervention efficacy and eventually the roles of acculturation and depression in smoking cessation for this particular ethnic group, as the levels of acculturation and depression might differ among smokers of different response patterns.

The MI-Fuzzy method provides a more sensitive statistical approach to characterizing smokers' response trajectory patterns by detecting subtle and graded effects of interventions in longitudinal studies with high-dimensional data and missing values [33-36]. Since heterogeneity is common in smokers' response to complex and longitudinal interventions, we hypothesized that distinct response trajectory patterns might exist but the number of patterns would be unknown a priori, and the patterns might be related to different cessation rates and background variables.

\section{Methods \\ Procedures}

The parent study is a two-arm parallel-group controlled clinical trial of a culturally adapted smoking cessation intervention conducted with a group of Korean American smokers $(N=109)$. They were randomized at a ratio of 1:1 to either a treatment arm or a comparison arm by opening a sealed envelope which contained a paper with a randomly assigned group number. The treatment arm received eight weekly 40-min individualized counseling sessions that incorporated 10 Koreanspecific cultural elements, whereas the comparison arm received eight weekly 10-min individualized counseling sessions that were not culturally adapted. Both arms also received active nicotine patches for 8 weeks from the target quit day. Due to the difference in the length of therapy session, participants were not blind to the treatment condition that they were assigned to. The study was guided by the theoretical framework of the Theory of Planned Behavior (TPB) [42, 43] and its three theoretical variables (attitudes, perceived social norms, and perceived behavioral control) were targeted by the smoking cessation intervention.

Irrespective of treatment condition, all participants received the same education about the deleterious effects of smoking on the human body and behavioral skills training to deal with nicotine withdrawal symptoms. In addition to this, the treatment arm received a culturally adapted cessation intervention focusing on culturespecific education and family coaching [12]. Participants in both arms had the same education on neurobiological changes in the brain associated with nicotine dependence and the treatment mechanism of nicotine replacement therapy. They received a 1-week supply of nicotine patches at each visit from the quit day for 8 weeks and returned used patches to be monitored for adherence. The medication was given with a gradual-dosage tapering schedule as follows: 21-mg dosage for 4 weeks, $14 \mathrm{mg}$ for 2 weeks and 7-mg for 2 weeks [12]. Quit day was set between the second and fourth therapy sessions of the individual therapy and each participant selected the day in consultation with the counselor.

The data of the present study were selected from 97 Korean American smokers after excluding 11 who did not participate in any follow-up assessments. The sample was comprised of 81 men and 16 women. Participants' ages ranged from 28 to 72 with an average of 49.8 (standard deviation $=9.2$ ).

\section{Measures}

Research questionnaires were written in Korean or in English and a bilingual research staff was present to assist participants if they needed help. The time spent to complete the questionnaires ranged from 30 to $60 \mathrm{~min}$. Participants were followed up over 1 year from the quit day and follow-up assessments were conducted at postquit $1,3,6$, and 12 months. Smoking status, nicotine dependence, and the three TPB variables (attitudes, perceived social norms and perceived behavioral control) were assessed at baseline and each of the four followups; one time during the smoking cessation interventions and three times after the interventions. All other variables including acculturation and depressive symptoms were assessed only at baseline.

Sociodemographic information was obtained on the following areas: gender, age, marital status, education, employment, acculturation, and length of residency in the United States. In this study, acculturation was measured in two ways: unidirectional (nine question items) and bidirectional (five question items). The unidirectional assessed changes in cultural orientation from Korean to American culture, whereas the bidirectional assessed the degree of cultural adaption in both Korean and American cultures [44].

History of smoking was assessed regarding age at which the participant began to smoke regularly, the average number of cigarettes smoked per day, any quit attempts made in the past year, and past use of cessation medications. Nicotine dependence was assessed using the Fagerström Test for Nicotine Dependence (FTND) [45]. The FTND consists of four dichotomous (e.g., do you find it difficult to refrain from smoking where it is forbidden) and two multiresponse items (e.g., how soon after you wake up do you smoke your fist cigarette?).

Alcohol use problems were assessed using the Alcohol Use Disorder Identification Test (AUDIT) [46]. The AUDIT consists of 10 items assessing the frequency and amount of alcohol use and items 1-3 assess individuals' 
alcohol consumption, items 4-6 examine abnormal drinking behavior, items 7-8 detect adverse psychological reactions, and items $9-10$ assess alcohol-related problems.

Depression was assessed using the Center for Epidemiologic Studies-Depression Scale (CESD-S) [47]. The CES-D used in this study is an adequate screening instrument for depressive disorder in the general population. The Korean version of the CES-D instrument was translated from the original CES-D and then validated with Koreans [48]. Instead of the cutoff point 16 recommended by Radloff, the cutoff point of 21 is considered as the best predictor of depression among Koreans [48].

Attitudes were assessed using the Perceived Risk and Benefits of Questionnaire (PRBQ) [49]. The PRBQ consists of 18 items for perceived risks of quitting (e.g., I will be less able to concentrate; and I will miss the taste of cigarettes) and 22 items for perceived benefits of quitting (e.g., I will smell cleaner; and I will feel proud that I was able to quit). Perceived social norms were assessed using the Perceived Social Norm Index [12]. This measure consists of two items regarding normative beliefs (e.g., I believe that my family or my friends wants me to quit smoking) and motivation to comply (e.g., I am willing to comply with the belief). Given that family and peers could have different norms toward quitting smoking, family and peer norms were assessed separately. For perceived behavioral control, we used the Self-Efficacy Scale that assessed the level of confidence in refusing smoking temptations at 10 high-risk situations (e.g., When I feel tense or anxious; and When I wake up in the morning) [50]. They were administered at baseline and at each of the four follow-up assessments (one time during the interventions and three times after the interventions).

Abstinence was defined as being continuously abstinent from the quit day except for the first 2-week grace period, which is referred to as prolonged abstinence. This definition, including the 2-week grace period, was based on the recommendation made by the Society for Research on Nicotine and Tobacco [51]. Self-reported abstinence was biochemically verified with expired-air $\mathrm{CO}(<6$ parts $\mathrm{ppm})$ and saliva cotinine $(\leq 30 \mathrm{ng} / \mathrm{ml})$ tests. We used a Micro + Smokerlyzer CO Monitor (Bedfont Scientific, NJ) and NicAlert ${ }^{\circ}$ test strips. The NicAlert ${ }^{\circ}$ test is a semi-quantitative measure of cotinine based on a colorimetric immunoassay reaction. A cutoff level of $20 \mathrm{ng} / \mathrm{ml}$ is generally used as an indicator of abstinence [52, 53]; hence, we used level $2(30-100 \mathrm{ng} / \mathrm{ml})$ as a cutoff level instead of level $1(10-30 \mathrm{ng} / \mathrm{ml})$. However, those who earned CO levels higher than $5 \mathrm{ppm}$ were all treated as smoking even if their saliva cotinine test yielded level 1 .

\section{Data analyses}

First, our pattern recognition model, multiple-imputationbased fuzzy clustering (MI-Fuzzy), was applied to identify cognitive and behavioral response patterns during and after the intervention. Second, we tested if acculturation and depression were associated with the identified patterns and abstinence. Also we explored whether gender would moderate the relationship between acculturation and abstinence and between depression and abstinence. Given two or more categorical variables (e.g., MIFuzzy derived patterns, gender) in the model, factorial logistic regression was appropriate and implemented in SAS 9.2 [54].

MI-Fuzzy [33-36, 55-58], a non-parametric unsupervised learning method, was specifically designed for characterizing longitudinal multi-component interventions and identifying differential response patterns resulting from known or unknown factors such as subjects' varied psychological reaction towards or engagement with the interventions using their observed scores on intervention attributes. MI-Fuzzy is the first clustering model to date that employs a full theoretical integration of (a) multiple imputation (MI), (b) fuzzy clustering, and (c) comprehensive validation [35, 36]. It simultaneously copes with real-world situations where smokers have a membership in multiple clusters, handles highdimensional longitudinal intervention data with missing values (e.g. multiple repeatedly-measured correlated constructs), and validates response patterns.

Briefly, MI-Fuzzy integrates MI techniques into the clustering to account for imputation uncertainty and uses "fuzzy degrees" to handle multiple membership situation, e.g., when clusters "touch" or "overlap", a single individual in a longitudinal smoking cessation study can have multiple memberships. This fuzzy degree score "summarizes" the smoker's response variation during the intervention, and determines one's membership in the cluster with one's highest degree score. It can handle non-normal and high-dimensional data with missing values and a mix of categorical and continuous variables, without prior assumptions of statistical distributions [35, 36]. To identify the optimal number of clusters, MIFuzzy includes a comprehensive validation process, graphs to visualize patterns generated from highdimensional data, MI-based fuzzy clustering index to validate response trajectory patterns, and statistical tests to examine clusters. Compared to typical clustering techniques such as hierarchical and K-means, MI-Fuzzy was demonstrated to have the best accuracy and consistency rates across imputed longitudinal datasets [35, 36]. MI-Fuzzy procedure developed in Matlab consists of three primary steps: Intervention attribute selection, MI-Fuzzy clustering, and cluster validation (see Appendix for further details). 
The difference in abstinence rates was examined among identified clusters. To examine the intervention attribute redundancy and over-fitting, we removed one category of intervention attributes at a time, re-ran the clustering, calculated the validation index, and then replaced the variables back into the model and iteratively repeated the process for each category of attributes. This strategy maximizes the information used to characterize the intervention process, identifies most important intervention components while minimizes model complexity. Sociodemographic information also was examined across the identified response patterns.

\section{Results}

There was no difference in any baseline characteristics when the two arms were compared before conducting MI-Fuzzy models. Three trajectory patterns were identified from our pattern recognition model MI-Fuzzy and named as culturally adapted (CA, $N=50)$, more bicultural (MB, $N=32)$ and less bicultural $(\mathrm{LB}, N=15)$ (Tables 1 and 2). The CA smokers were those from the treatment arm with the culturally adapted cessation intervention, while $\mathrm{MB}$ and $\mathrm{LB}$ groups were from the comparison arm with the standard cessation intervention. Interestingly, response heterogeneity was within the comparison arm rather than the treatment arm.

As shown in Fig. 1, three optimal clusters were revealed by the abrupt low value of $\mathrm{XB}_{\mathrm{m}}$ at three clusters. Sammon mapping (Fig. 2) further supported three clusters, where asterisks represent the projected centroids and dots represent subjects within the identified clusters. The values on the two axes are the projected normalized scores for these subjects. Our model also identified Perceived Benefits of Quitting and Perceived Family Norm for Quitting as two most important variables for this culturally adapted cessation intervention in our attribute redundancy test, because adding other attributes dramatically decreased the accuracy rates and failed the validity and visualization tests $[35,36]$. Figure 3 (x-axis: months in the intervention; $y$-axis: scores on the Perceived Benefits or on the Perceived Family Norm) displays the overall trajectory patterns of these three

Table 1 Differences in intervention attributes among three identified patterns

\begin{tabular}{|c|c|c|c|c|c|c|}
\hline Intervention Attributes ${ }^{a}$ & $\begin{array}{l}\text { Culturally Adapted } \\
\text { M }\end{array}$ & $\begin{array}{l}N=50 \\
S E^{b}\end{array}$ & $\begin{array}{l}\text { More Bicultural } \\
\text { M }\end{array}$ & $\begin{array}{l}N=32 \\
\mathrm{SE}^{2}\end{array}$ & $\begin{array}{l}\text { Less Bicultural } \\
\text { M }\end{array}$ & $\begin{array}{l}N=15 \\
S E^{2}\end{array}$ \\
\hline \multicolumn{7}{|l|}{ Included in MI-Fuzzy } \\
\hline Perceived benefit at $1-\mathrm{M} \mathrm{F/U^{*** }}$ & 133.56 & 1.88 & 135.41 & 2.33 & 112.91 & 5.67 \\
\hline Perceived benefit at 3-M F/U & 128.72 & 3.05 & 125.19 & 4.72 & 113.21 & 4.06 \\
\hline Perceived benefit at 6-M F/U** & 129.23 & 2.30 & 135.05 & 2.23 & 115.00 & 4.32 \\
\hline Perceived benefit at $12-\mathrm{M} F / \mathrm{U}^{* * *}$ & 129.50 & 3.16 & 135.65 & 2.65 & 113.79 & 4.98 \\
\hline 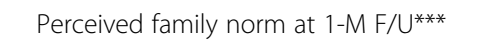 & 5.63 & 0.14 & 5.57 & 0.10 & 3.91 & 0.31 \\
\hline Perceived family norm at 3-M F/U*** & 5.59 & 0.09 & 5.57 & 0.11 & 4.21 & 0.28 \\
\hline 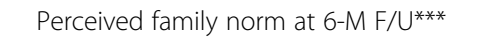 & 5.36 & 0.14 & 5.45 & 0.11 & 4.08 & 0.30 \\
\hline Perceived family norm a4 12-M F/U*** & 5.14 & 0.16 & 5.43 & 0.12 & 3.79 & 0.24 \\
\hline \multicolumn{7}{|l|}{ Excluded } \\
\hline Perceived risk at 1-M F/U** & 56.00 & 2.16 & 53.07 & 2.70 & 72.50 & 6.50 \\
\hline Perceived risk at 3-M F/U* & 64.46 & 2.26 & 54.48 & 2.34 & 75.13 & 4.87 \\
\hline Perceived risk at 6-M F/U & 64.61 & 2.84 & 67.76 & 3.15 & 77.71 & 5.15 \\
\hline Perceived risk at 12-M F/U & 63.10 & 2.75 & 68.43 & 3.09 & 80.40 & 4.65 \\
\hline Perceived peer norm at 1-M F/U & 3.72 & 0.33 & 3.93 & 0.42 & 2.42 & 0.82 \\
\hline Perceived peer norm at 3-M F/U* & 3.70 & 0.38 & 4.48 & 0.32 & 1.93 & 0.64 \\
\hline Perceived peer norm at 6-M F/U & 3.68 & 0.34 & 4.14 & 0.39 & 2.29 & 0.47 \\
\hline Perceived peer norm at 12-M F/U & 4.00 & 0.32 & 3.57 & 0.53 & 1.73 & 0.73 \\
\hline Self-efficacy at 1-M F/U*** & 40.94 & 1.01 & 40.41 & 1.16 & 32.58 & 2.00 \\
\hline Self-efficacy at 3-M F/U & 37.54 & 1.31 & 39.43 & 1.54 & 34.60 & 1.99 \\
\hline Self-efficacy at 6-M F/U & 38.34 & 1.70 & 35.68 & 1.61 & 31.93 & 2.05 \\
\hline Self-efficacy at 12-M F/U & 36.90 & 1.44 & 34.74 & 1.98 & 29.40 & 2.38 \\
\hline
\end{tabular}

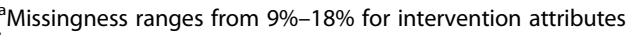

${ }^{b}$ For multiple imputation, the means and standard errors were computed based on ten imputed data sets (Robin, 1996; Shafer, 1997) $M$ Month, $F / U$ follow-up

${ }^{*} p<.05 ;{ }^{* *} p<.01 ;{ }^{* * *} p<.001$ 
Table 2 Background difference among three identified clusters

\begin{tabular}{|c|c|c|c|c|c|c|}
\hline \multirow[t]{2}{*}{ Variable $^{a}$} & \multirow{2}{*}{$\begin{array}{l}\text { Culturally Adapted } \\
\text { M }\end{array}$} & \multirow{2}{*}{$\begin{array}{l}N=50 \\
\mathrm{SD}\end{array}$} & \multirow{2}{*}{$\begin{array}{l}\text { More Bicultural } \\
\text { M }\end{array}$} & \multirow{2}{*}{$\begin{array}{l}N=32 \\
S D\end{array}$} & \multirow{2}{*}{$\begin{array}{l}\text { Less Bicultural } \\
\text { M }\end{array}$} & \multirow{2}{*}{$\begin{array}{l}N=15 \\
S D\end{array}$} \\
\hline & & & & & & \\
\hline Age & 50.08 & 8.18 & 50.47 & 8.37 & 45.47 & 13.38 \\
\hline Education & 14.74 & 2.49 & 14.97 & 1.71 & 14.13 & 1.96 \\
\hline Bidirectional Acculturation** & 2.83 & 0.37 & 3.18 & 0.41 & 3.02 & 0.52 \\
\hline Smoking duration & 18.32 & 8.66 & 19.25 & 9.03 & 16.13 & 8.72 \\
\hline Age at smoking onset & 19.76 & 4.03 & 20.75 & 4.17 & 19.20 & 3.75 \\
\hline Cigarettes per day at baseline & 16.58 & 5.44 & 16.88 & 4.82 & 17.60 & 7.91 \\
\hline Cigarettes per day at $12-\mathrm{M} \mathrm{F} / \mathrm{U}^{*}$ & 4.88 & 6.47 & 8.22 & 7.40 & 9.36 & 6.10 \\
\hline Nicotine dependence at 1-M F/U** & 0.10 & 0.52 & 0.14 & 0.35 & 1.00 & 2.05 \\
\hline Nicotine dependence at 3-M F/U & 0.98 & 1.93 & 0.52 & 1.25 & 1.14 & 1.79 \\
\hline Nicotine dependence at 6-M F/U & 1.36 & 2.26 & 1.68 & 2.21 & 2.15 & 1.86 \\
\hline Nicotine dependence at12-M F/U & 1.40 & 2.07 & 2.35 & 2.35 & 2.50 & 2.31 \\
\hline \multirow[t]{2}{*}{ Alcohol use at baseline } & 5.70 & 5.74 & 4.97 & 4.66 & 5.93 & 6.25 \\
\hline & $\mathrm{N}$ & $(\%)$ & $\mathrm{N}$ & (\%) & $\mathrm{N}$ & $(\%)$ \\
\hline Female & 8 & 8.25 & 5 & 5.15 & 3 & 3.09 \\
\hline Marital status (Married)* & 42 & 43.30 & 27 & 27.84 & 8 & 8.25 \\
\hline Employment (Yes) & 45 & 46.39 & 29 & 29.90 & 13 & 13.40 \\
\hline Alcohol use problems (Yes) & 19 & 19.59 & 10 & 10.31 & 4 & 4.12 \\
\hline
\end{tabular}

${ }^{a}$ The 11 smokers who did not participate this study did not differ from those included for analyses across all these variables (ps:0 .11-.98) $M$ Month, $F / U$ follow-up

${ }^{*} p<.05 ;{ }^{* *} p<.01 ;{ }^{* * *} p<.001$

clusters for the Perceived Benefits and Perceived Family Norm. These visual results further reinforce the quantitative results that indicate two clusters exist within the comparison arm.

Table 1 displays significance levels of the differences among the three identified patterns with two included

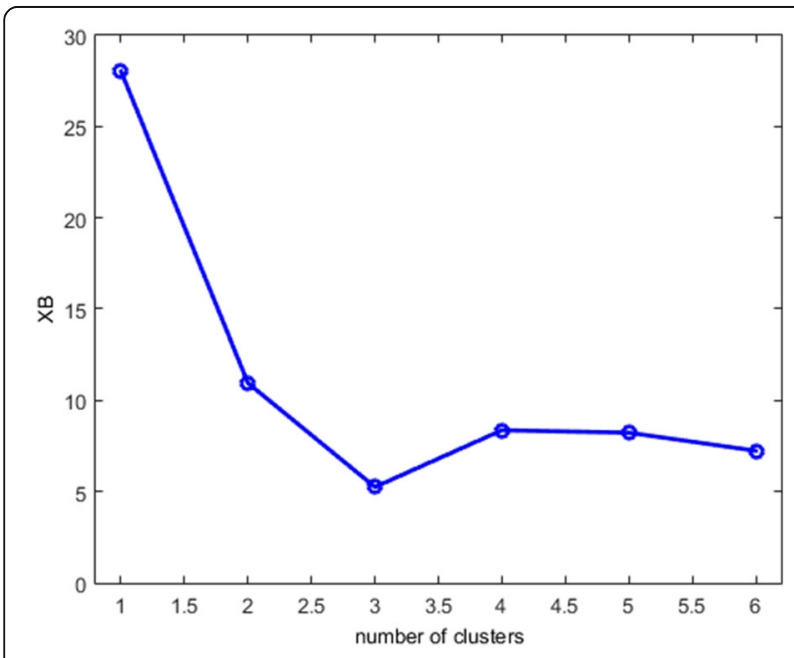

Fig. 1 MIFuzzy validation: the minimum $X B_{m i}$ index for the optimal 3 clusters, legend: Optimal 3 clusters with abrupt decreased or minimal value of $X B$ validation index. $X$-axis: the number of clusters; $Y$-axis: the values of $X B$ attributes (the Perceived Benefits and Perceived Family Norm) and other excluded attributes. Overall, the differences were between the CA and LB groups, or between the MB and LB groups, but similar between the CA and $M B$ groups. The $C A$ and $M B$ groups differed from the LB group in marital status and the MB group differed

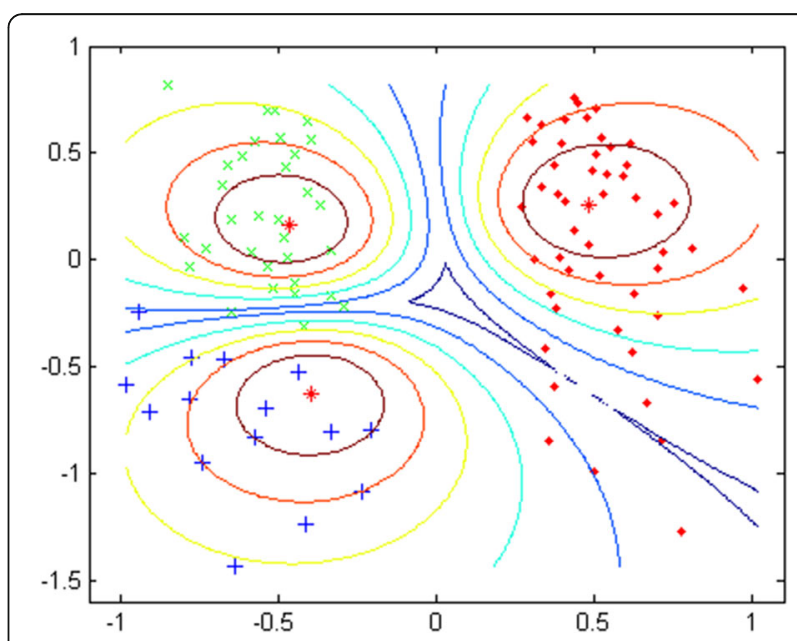

Fig. 2 Sammon Mapping of three latent clusters, legend: Sammon mapping further supported three latent clusters, where asterisks represent the projected centroids and dots represent subjects within the identified clusters. The values on the two axes are the projected normalized scores for these subjects 

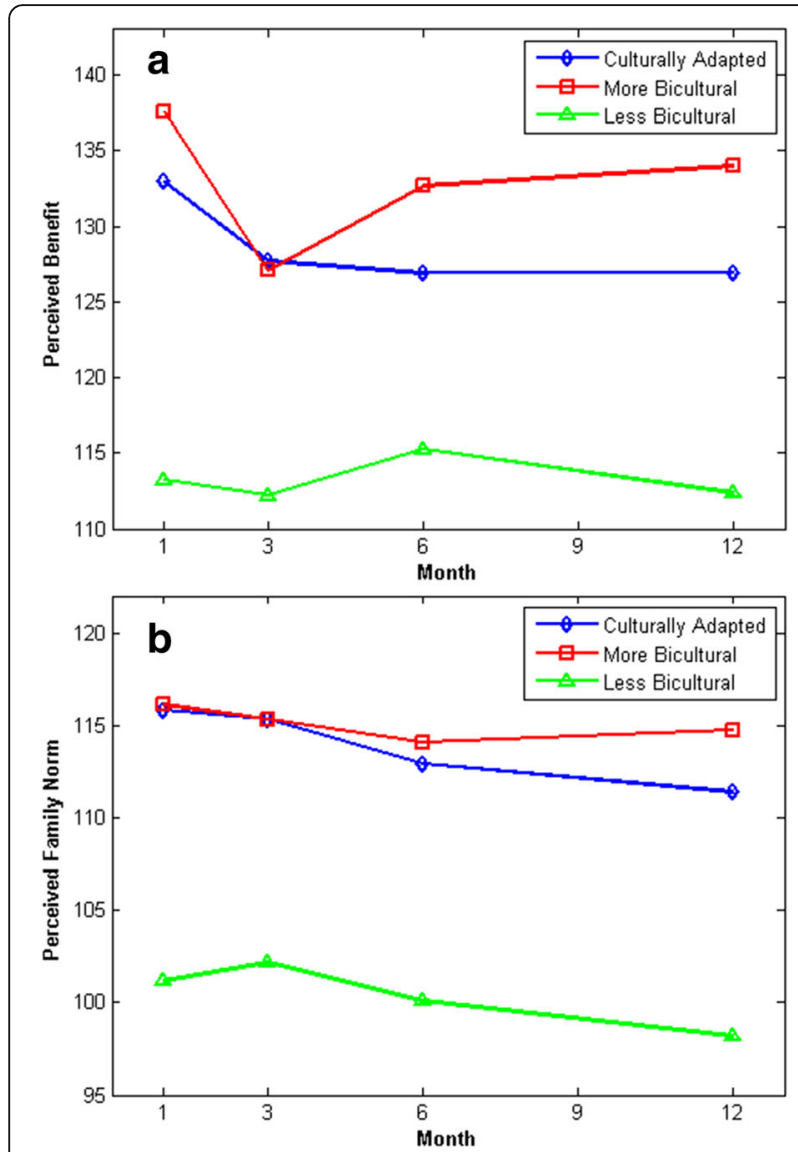

Fig. 3 (a) Perceived Benefit Trajectory Patterns, (b) Perceived Family Norm Trajectory Patterns. Identified Trajectory Patterns for CulturallyAdapted (blue), more bicultural (MB, red) and less bicultural (LB, green) groups across included intervention components; $X$-axis: the number of intervention months; Y-axis: (a) Perceived Benefit or (b) Perceived Family Norm

from the CA and LB groups in bidirectional acculturation. Except for these two, the three groups were comparable on most baseline variables (see Table 2). This lack of difference among the three response patterns reinforces that clustering these smokers based on routine background variables likely would not be useful to uncover much heterogeneity in treatment responses.

Associated with the three patterns, three levels of depression were detected: near-low for CA (mean/standard error [M/SE]: 8.60/1.29); low for MB (M/SE: 5.94/1.34); and high for LB (M/SE: 18.67/4.34; $\mathrm{p}<.01)$. Both Bonferroni and Tukey pairwise tests indicated the depression levels differed between $C A$ and $L B$ or between $M B$ and LB. The high-depressed LB group had a 1.03\% abstinence rate, while the low-depressed MB group had a 5.15\% abstinence rate, and the near-low-depressed CA group achieved the highest rate of $21.65 \%$. Neither marital status nor depression had a direct effect on abstinence; however, the near-low depressed CA group significantly differed from the high-depressed LB group at the 12-month prolonged abstinence (odds ratio: 10.138, SE: $0.43, \mathrm{p}<0.01$ ). Neither significant difference was detected in abstinence rates between the $\mathrm{MB}$ and $\mathrm{LB}$ groups, nor significant interactions found between these groups and depression ( $p>.65$ ) or marital status ( $p>$.99). Gender did not moderate the relationship between depression and abstinence rates $(\mathrm{p}=0.24)$, or between acculturation and abstinence rates $(\mathrm{p}=0.58)$, which could be attributable to the small number of female smokers in this study.

\section{Discussion}

Our MI-Fuzzy model identified three distinctive response patterns among Korean American smokers who received either a culturally adapted or standard cessation intervention. It is interesting to note that Korean Americans in the treatment arm manifested a homogenous response pattern (the Culturally Adaptive, CA group), whereas those in the comparison arm responded with two distinctive patterns (the More Bicultural, MB; and Less Bicultural, LB groups). The LB and MB groups received the same standard cessation intervention and thus, at first glance, it was not clear why the two groups manifested such different response patterns.

With our further analyses, we found the LB group was less likely to be married than the other two groups, which might have affected the former group's unique response pattern compared to the other groups. Perceived family norm for quitting was an important intervention attribute differentiating the LB group from the others. A separate mediation analysis identify that the variable was the only significant mediator of the cessation intervention on abstinence in this study [12]. Given this, those who were married might have perceived a stronger family norm favoring quitting than those who were not married and have manifested a similar response pattern to the cessation intervention. The finding is also in support of the report that home smoking restriction and social network discouraging smoking as correlates of smoking cessation [15]. It could be assumed that those who were married were more likely to have home smoking bans by their non-smoking partners than those who lived alone. A smoking cessation study conducted in China also reported that married smokers were more likely to achieve abstinence than their counterparts [59].

The MB group differed from the LB and CA groups in bidirectional acculturation although no difference was found in unidirectional acculturation. Compared to the other two, the MB group was more bicultural, meaning that the group was familiar with both Korean and American cultures. It was found that bicultural Korean men were less likely to be current smokers while bicultural and acculturated Korean women were more likely to be current smokers than their traditional counterparts [14]. The 
majority of participants in this study were men and thus, the bicultural MB group might have endorsed greater perceived benefits of quitting and perceived family norm for quitting than the LB group despite the fact that both received the same standard cessation intervention.

Current depressive symptoms differed between the LB and the other two response-pattern groups although the symptoms did not have a direct effect on abstinence. Interestingly, the high-depressed LB group achieved a significantly lower abstinence rate than the near-lowdepressed CA group. This finding is supportive of the notion that smokers who have higher levels of current depressive symptoms are less likely to quit $[17,23]$. It is interesting, however, abstinence rates between the $\mathrm{MB}$ and LB groups were not statistically different. This finding may suggest that Korean Americans, irrespective of their levels of acculturation and depression, require a more intensive and culturally adapted cessation intervention for a successful cessation outcome.

Gender did not moderate the relationship between depression and abstinence. Similarly, there was no gender interaction effect with acculturation on abstinence. These findings might be related to the small sample of women in this study. Larger clinical trials should be conducted to verify the role of gender in this particular Asian ethnic subgroup. In addition, the study is limited because depression was not determined by the Structured Clinical Interview [60] but by the CES-D. Nevertheless, the Korean version of the CES-D is an excellent screening tool for depression with its cutoff point of 21 for Koreans [48]. This cut-off score is higher than in the original CES-D because Koreans have been found to give negative responses for positive effects, thereby increasing their scores for depression even when they may not be depressed.

The present study has several limitations in regards to the generalizability of the findings. First, the sample size was designed for Korean smokers who are a small population in the United States; particularly the number of female smokers was relatively small compared to male smokers. Therefore, the study may not be able to infer findings to the general U.S. population or other minority populations of a different cultural background. However, the idea of our deep culturally adapted intervention design and implementation may be tailored to other minority populations. Second, participation in the study was limited to those who had smoked at least 10 cigarettes per day. Thus, findings might not be applicable to those who smoke fewer.

\section{Conclusions}

The present study identified three patterns of treatment responses to a smoking cessation intervention that was conducted with Korean Americans. Marital status, acculturation, and depression might have contributed to the difference in treatment responses between the $\mathrm{MB}$ and $\mathrm{LB}$ pattern groups in the comparison arm. Although findings are preliminary, it appeared that the culturally adapted cessation intervention appeared effective for Korean American smokers regardless of their marital status, acculturation, and depression. Conversely, those who received a standard cessation intervention did poorly irrespective of the characteristics. These findings were new from the present study, whereas the parent study did not find any relationship between baseline characteristics, including acculturation and depression, and treatment outcomes. A culturally adapted intervention should target the low-bicultural and high-depressed LB group to assist for their successful smoking cessation.

\section{Appendix}

MI-Fuzzy procedure developed in Matlab consists of three primary steps: intervention attribute selection, MIFuzzy clustering, and cluster validation. Each of them is described below.

\section{Intervention attribute selection}

Intervention attributes in MI-Fuzzy were based on data availability and selected to maximize information that depicts individual response variations resulting from their psychological factors and engagement with the intervention. All designed intervention components and time for cessation counseling were included at the beginning. The three components were (a) cognitive behavioral therapy, (b) cultural adaptation, and (c) nicotine replacement therapy. The first two components were psychological reactions to the culturally adapted cognitive behavioral therapy, which were measured by scores on Perceived Risks and Benefits of Quitting Smoking, Perceived Family and Peer Norm for Quitting, and Self-efficacy in Quitting scales. Each scale has four repeated measures collected at four follow-ups: 1, 3, 6, and 12 months from the quit day, total 20 intervention attributes (5 subscales" 4 times). The last component 'nicotine replacement therapy' was measured by the number of nicotine patches returned after use ( 1 attribute) and the counseling time was measured in the unit of minutes (1 attribute). In all, 22 intervention attributes were initially used for MI-Fuzzy clustering (Table 1).

\section{MI-Fuzzy clustering}

Unlike typical clustering models and other missing data imputing methods (e.g., mean, regression, and hot deck) that introduce bias and lose precision, MI-Fuzzy iteratively implements the multiple-imputation based fuzzy clustering procedure using all intervention attributes to account for imputation uncertainty and ensure pattern stability and consistency. With high-dimensional data, when a 
smoker has a missing value on an attribute, MI repeatedly draws information from several other available attributes to impute the informative missing value. Our data (ranges from $9 \%$ to $18 \%$ missingness on each of tested intervention attribute; $65 \%$ participants have all values, $34 \%$ have more than 5 values on intervention attributes) follow an arbitrary missing pattern and 10 imputed datasets were generated using the Markov Chain Monte Carlo method with multiple chains, non-informative Jeffreys prior of the Bayesian approach, and 500 burn-in iterations. For each imputed data set, we minimized this fuzzy objective function (e.g., we minimized the intra-cluster variance). Given a termination clustering number $\left(\mathrm{C}_{\mathrm{T}}\right)$ of $7\left[\mathrm{C}_{\mathrm{T}}=(\mathrm{N} / 2)^{1 / 2}\right]$ where $\mathrm{N}$ is the sample size, the MI-Fuzzy algorithm searched for the optimal number of clusters through a comprehensive validation procedure.

\section{Pattern validation}

MI-Fuzzy validataion procedure includes MI-based clustering inconsistency and accuracy rate; validation indices; high-dimensional data mapping and trajectory pattern visualization; and statistical testing of intervention attributes. The MI-based clustering inconsistency rate was calculated as the number of cases with inconsistent labels across imputed data sets divided by the total sample size (smaller is better, as larger values indicate instability); and the MI-based clustering accuracy rate was computed as the average fraction of correctly-classified cases across imputed data sets (larger is better). The MI-based Xie-Beni Index $\left(\mathrm{XB}_{\mathrm{mi}}\right)$, widely used for fuzzy clustering, quantifies the ratio of the total variation within and between clusters, with smaller being better. High-dimensional data mapping based on Sammon mapping was incorporated into the algorithm to visualize the clusters in 2-dimensional space, while the trajectories for repeatedly measured intervention attributes reflect the intensity of response variation for each cluster.

The newly developed MI-Fuzzy achieved the highest accuracy rate of $100 \%$, while the accuracy rates range from below $50 \%$ to $93.81 \%$ for K-Means, Self-organizing Map, Gaussian Mixture, Bayes, and Hierarchical clustering. Across the imputed datasets, MI-Fuzzy also achieved the lowest inconsistency rate of $6 \%$, while others yielded $10-50 \%$ rates of inconsistency. These results are consistent with our previous findings in two observational studies $[35,36]$.

\section{Acknowledgements}

We appreciate participants who were available at all four follow-up assessments of the parent study.

\section{Funding}

This work was supported by the Natinal Institute on Drug Abuse ([NIDA] R01DA033323-01A1 to Dr. Fang) and the pilot study award from National Center for Advancing Translational Sciences under Grant 5UL1TR000161-04 to Dr. Fang and partially by the (NIDA, 5K23DA021243-02 to Dr. Kim).
Availability of data and materials

Data and materials are available upon request.

\section{Authors' contributions}

SK wrote background and methods (participants and study measures) and reviewed the manuscript. HF and ZZ conducted data analyses. HF. wrote and reviewed MIFuzzy background, methods (data analyses), results and the manuscript. SK and HF jointly wrote Discussion and Conclusion. KB, JD, DZ. and JA reviewed drafts of the manuscript and provided critical feedback. All authors contributed to and approved the final manuscript.

\section{Ethics approval and consent to participate}

The parent study was approved by the institutional review board of University of Massachusetts Medical School.

\section{Consent for publication}

Not Applicable.

\section{Competing interests}

The authors' declare that they have no competing interests.

\section{Publisher's note}

Springer Nature remains neutral with regard to jurisdictional claims in published maps and institutional affiliations.

\section{Author details \\ ${ }^{1}$ University of Massachusetts, Boston, Boston, MA 02125, USA. ${ }^{2}$ University of Massachusetts Dartmouth and Medical School Dartmouth, Dartmouth, MA 02747, USA. ${ }^{3}$ Hunter College, City University of New York, New York, New York 10010, USA. ${ }^{4}$ Department of Computer and Information Science, College of Engineering, University of Massachusetts Dartmouth, Dion Building, Room 317285 Old Westport Road Dartmouth, Dartmouth, MA 02747-2300, USA. ${ }^{5}$ Division of Biostatistics and Health Services Research Department of Quantitative Health Sciences, University of Massachusetts Medical School, Albert Sherman Bldg, Office: AS8-2061, 368 Plantation St. Worcester, Dartmouth, MA 01605-0002, USA. 'University of California San Diego, Deparetment of Psychiatry, 9500 Gilman Drive \#0602, La Jolla, CA 92093-0602, USA.}

Received: 28 July 2016 Accepted: 6 July 2017

Published online: 24 July 2017

\section{Reference}

1. U.S. Department of Health and Human Services. The health consequences of smoking - 50 years of progress. A report of the surgeon general: executive summary. Rockville: DPHH Services, Office of the Surgeon General; 2014.

2. University of California Los Angeles Center for Health Research Policy. (2016). California, health interview survey: adult survey, 2001-2015. Los Angeles, CA: UCLA. 2016. http://healthpolicy.ucla.edu/chis/about/Pages/ about.aspx. Accessed 3 Mar 2017.

3. Leistikow BN, Chen M, Tsodikov A. Tobacco smoke overload and ethnic, state, gender, and temporal cancer mortality disparities in Asian-Americans and Pacific islander-Americans. Prev Med. 2006;42:430-4.

4. Centers for Disease Control and Prevention. Smoking-attributable mortality, years of potential life lost, and productivity losses — United States, MMWR. 2008;57(45):1226-1228.2000-2004.

5. Caraballo RS, Yee SL, Groerer J, Mirza SA. Adult tobacco use among racial and ethnic groups living in the United States, 2002-2005. Public Health Research, Practice and Policy. 2008;5(3):1-9.

6. Brugge D, Dejong W, Hyde J, Le Q, Shih CS, Wong A, Tran A. Development of targeted message concepts for recent Asian immigrants about secondhand smoke. J Commun Health. 2002;7:25-37.

7. Liu JJ, Wabnitz C, Davidson E, Bhopal RS, White M, Johnson MR, Netto G, Sheikh A. Smoking cessation interventions for ethnic minority groups-a systematic review of adapted interventions. Prev Med. 2013;57:765-75.

8. Perez-Stable EJ, Marin G, Posner SF. Ethnic comparison of attitudes and beliefs about 20 cigarette smoking. J Gen Intern Med. 1998;13:167-74.

9. Fang CY, Ma GX, Miller SM, Tan Y, Su X, Shive S. A brief smoking cessation intervention for Chinese and Korean American smokers. Prev Med. 2006;43:321-4. 
10. McDonnell DD, Kazinets Z, Lee HJ, Moskowitz JM. An internet-based smoking cessation program for Korean Americans: results from a randomized controlled trial. Nicotine Tob Res. 2011;13:336-43.

11. Zhu S-H, Cummins SE, Wong S, Gamst AC, Tedeschi GJ, Reyes-Nocon J. The effects of a multilingual telephone quitline for Asian smokers: a randomized controlled trial. J Natl Cancer Inst. 2012;104:299-310.

12. Kim SS, Kim SH, Fang H, Kwon S, Shelley D, Ziedonis DM. A culturally adapted smoking cessation intervention for Korean Americans: a mediating effect of perceived family norm toward quitting. J Immigr Minor Health. 2015:17:1120-9.

13. Kim SS, Ziedonis D, Chen K. Tobacco use and dependence in Asian Americans: a review of the literature. Nicotine Tob Res. 2007;9(2):169-84.

14. Lee S, Sobal J, Frongillo EA. Acculturation and health in Korean Americans. Soc Sci Med. 2000;51:159-73.

15. Ji M, Hofstetter RC, Hovell M, et al. Smoking cessation patterns and predictors among adult Californians of Korean descent. Nicotine Tob res. 2005:7(1):59-69.

16. Brown SA, Vik PW, Patterson TL, Grant I, Schuckit MA. Stress, vulnerability and adult alcohol relapse. J Stud Alcohol Drugs. 1995;56:538-45.

17. McClave AK, Dube SR, Strine TW, Kroneke K, Caraballo RS, Mokdad AH. Associations between smoking cessation and anxiety and depression among U.S. adults. Addict Behav. 2009;34:491-7.

18. Tsoh JY, Lam JN, Delucchi KL, Hall SM. Smoking and depression in Chinese Americans. American Journal of Medical Science. 2003;326:187-91.

19. Wiecha J, Lee $V$, Hodgkins J. Patterns of smoking, risk factors for smoking, and smoking cessation among Vietnamese men in Massachusetts (United States). Tob Control. 1998;7:27-34.

20. Hitsman B, Papandonatos GD, McChargue DE, DeMott A, Herrera MJ, Spring B, Borrelli B, Niaura R. Past major depression and smoking cessation outcome: a systematic review and meta-analysis update. Addiction. 2013; 108:294-306.

21. Weinberger AH, Pilver CE, Desai RA, Mazure CM, McKee SA. The relationship of major depressive disorder and gender to changes in smoking for current and former smokers: longitudinal evaluation in the U.S. population. Addiction. 2012;107:1847-56.

22. Zvolensky MJ, Farris SG, Leventhal AM, Ditre JW, Schmidt MB. Emotional disorders and smoking: relations to quit attempts and cessation strategies among treatment-seeking smokers. Addict Behav. 2015;40:126-31.

23. Pomerleau OF, Pomerleau CS, Mehringer AM, Snedecor SM, Ninowski R, Sen A. Nicotine dependence, depression, and gender: characterizing phenotypes based on withdrawal discomfort, response to smoking, and ability to abstain. Nicotine Tob Res. 2005;7:91-102.

24. Husky MM, Mazure CM, Paliwal P, McKee SA. Gender differences in the comorbidity of smoking behavior and major depression. Drug Alcohol Depen. 2008:93:176-9.

25. Poulin C, Hand D, Boudreau B, Santor D. Gender differences in the association between substance use and elevated depressive symptoms in a general adolescent population. Addiction. 2005;100:525-35.22.

26. Luk JW, Tsoh JY. Moderation of gender on depression and smoking in Chinese Americans. Addict Behav. 2010;35:1040-3.

27. Yun WJ, Shin MH, Kweon SS, Rhy SY, Rhee JA. Association of smoking status, cumulative smoking, duration of smoking cessation, age of starting smoking, and depression in Korean adults. BMC Public Health. 2012:12:724

28. Kim SS, Kim SH, Seward G, Fortuna L, McKee S. Korean American women's experiences with smoking and factors associated with their quit intentions. ISRN Addiction. 2013; doi:10.1155/2013/796570.

29. Park MB, Kim C-B, Nam EW, Hong KS. Does South Korea have hidden female smokers: discrepancies in smoking rates between self-reports and urinary cotinine level. BMC Womens Health. 2014;14:156.

30. Borrelli $B$, Bock $B$, King $T$, Pinto B, Marcus BH. The impact of depression on smoking cessation in women. Am J Prev Med. 1996;12:378-87.

31. Leventhal AM, Waters AJ, Boyd S, Moolchan ET, Lerman C, Pickworth WB. Gender differences in acute tobacco withdrawal: effects on subjective, cognitive, and physiological measures. Exp Clin Psychopharm. 2007;15: 21-36.

32. Xu J, Azizian A, Monterosso J, Domier CP, Brody AL, Fong TW, London ED. Gender effects on mood and cigarette craving during early abstinence and resumption of smoking. Nicotine Tob Res. 2008;10:1653-61.

33. Zhang Z, Fang $H$, Wang $H$. A new Ml-based visualization aided validation index for mining big longitudinal web trial data. IEEE Access. 2016:4:2272-80. doi:10.1109/ACCESS.2016.2569074.23.
34. Zhang $Z$, Fang $H$, Wang $H$. Multiple imputation based clustering validation (MIV) for big longitudinal trial data with missing values in e-health. J Med Syst. 2016;40(6):1-9.

35. Fang $H_{\text {, Johnson }}$, Stopp C, Espy KA. A new look at quantifying tobacco exposure during pregnancy using fuzzy clustering. Neurotoxicol Teratol. 2011;33:155-65.

36. Fang H, Dukic V, Pickett KE, Wakschlag L, Espy KA. Detecting graded exposure effects: a report on an East Boston pregnancy cohort. Nicotine Tob Res. 2012;14:1115-20.

37. Kent D, Hayward RA. Limitations of applying summary results of clinical trials to individual patients. JAMA. 2011;298:1209-12.

38. Sculpher M. Subgroups and heterogeneity in cost-effectiveness analysis. PharmacoEconomics. 2008;26:799-806.

39. Anderson KG, Ramo DE, Brown SA. Life stress, coping and comorbid youth: an examination of the stress-vulnerability model for substance relapse. Psychoactive Drugs. 2006;38:255-62.

40. Dagher A, Tannenbaum B, Hayashi T, Pruessner JC, McBride D. An acute psychosocial stress enhances the neural response to smoking cues. Brain Res. 2009:1293:40-8.

41. Schoedel KA, Hoffmann EB, Rao Y, Sellers EM, Tyndale RF. Ethnic variation in CYP2A6 and association of genetically slow nicotine metabolism and smoking in adult Caucasians. Pharmacogenetics. 2004;14:615-26.

42. Ajzen I. Attitudes, personality, and behavior. Chicago: Dorsey; 1988.

43. Aizen I. The theory of planned behavior. Organ Behav Hum Decis Process. 1991;50:179-211.

44. Suinn RM, Ahuna C, Khoo G. The Suinn-Lew Asian self-identity acculturation scale: concurrent and factorial validation. Educ Psychol Meas. 1992;52:1041-6.

45. Heatherton TF, Kozlowski LT, Frecker RC, Fagerström KO. The Fagerström test for nicotine dependence: a revision of the Fagerström tolerance questionnaire. Brit J Addict. 1991;86:1119-27.

46. Babor TF, Higgins-Biddle JC, Saunders JB, Monteiro MG. The alcohol use disorders identification test: guidelines for use in primary care. 2nd ed. Geneva: World Health Organization; 2001.

47. Radloff LS. The CES-D scale: a self-report depression scale for research in the general population. Appl Psychol Meas. 1977;1:385-401.

48. Cho MJ, Kim KH. Use of the Center for Epidemiologic Studies Depression (CES-D) scale in Korea. J Nerv Ment Dis. 1998:186:304-10.

49. McKee SA, O'Malley SS, Salovey P, Krishnan-Sarin S, Mazure CM. Perceived risks and benefits of smoking cessation: gender-specific predictors of motivation and treatment outcome. Addict Behav. 2005;30:423-35.

50. Kim SS, Kim SH, Gulick EE. Cross-cultural validation of smoking abstinence self-efficacy scale with Korean-American men. Issues Ment Health Nurs. 2009;30:122-30.

51. Hughes JR, Keely JP, Niaura RS, Ossip-Klein DJ, Richmond RL, Swan GE. Measures of abstinence in clinical trials: issues and recommendations. Nicotine Tob Res. 2003:5:13-25.

52. Murray RP, Connett JE, Buist AS, Gerald LB, Eichenhorn MS. Experience of black subjects in the lung health study smoking cessation intervention program. Nicotine Tob Res. 2001;3:375-82.

53. Voorhees CC, Stillman FA, Swank RT, Heagerty PJ, Levine DM, Becker DM. Heart, body, and soul: impact of church-based smoking cessation interventions on readiness to quit. Prev Med. 1996;25:277-85.

54. SAS Institute Inc. The GLIMMIX procedure. In: SAS/STAT ${ }^{\oplus} 9.2$ User's Guide. Cary: the Author; 2013.

55. Fang H, Espy KA, Rizzo ML, Stopp C, Wiebe CA, Stroup WW. Pattern recognition of longitudinal trial data with nonignorable missingness: an empirical case study. Int J Inf Technol Decis Mak. 2009:8(3):491-513.

56. Fang H, Rizzo ML, Wang H, Espy KA, Wang Z. A new nonlinear classifier with a penalized signed fuzzy measure using effective genetic algorithm. Pattern Recogn. 2010;43(4):1393-401.

57. Fang $H$, Zhang Z. An enhanced visualization method to aid behaviora trajectory Patten recognition infrastructure for big longitudinal data. IEEE Trans Big Data. 2017; doi:10.1109/TBDATA.2017.2653815.

58. Fang H. MIFuzzy clustering for incomplete longitudinal data in smart health. Smart Health. 2017; doi:10.1016/j.smhl.2017.04.002.

59. Sun HQ, Guo S, Chen DF, et al. Family support and employment as predictors of smoking cessation success: a randomized, double-blind, placebo-controlled trial of nicotine sublingual tablets in Chinese smokers. Am J Drug Alcohol Ab. 2009;35:183-8.

60. Williams JB, Gibbon M, First MB, et al. The structured clinical interview for DSMIII-R (SCID). II. Multisite test-retest reliability. Arch Gen Psychiat. 1992;49:630-6. 